\title{
Trait probability density (TPD): measuring functional diversity across scales based on TPD with $\mathrm{R}$
}

\author{
Carlos P. Carmona, ${ }^{1,6}$ Francesco de Bello, ${ }^{2,3}$ Norman W. H. Mason, ${ }^{4}$ and Jan Lepś 2,5 \\ ${ }^{1}$ Institute of Ecology and Earth Sciences, University of Tartu, Lai 40, Tartu 51005 Estonia \\ ${ }^{2}$ Department of Botany, Faculty of Science, University of South Bohemia, Branišsovská 31, České Budejovice 37005 Czech Republic \\ ${ }^{3}$ Centro de Investigaciones sobre Desertificacion (CSIC-UV-GV), Carretera Moncada-Náquera Km 4.5, Moncada, Valencia 46113 Spain \\ ${ }^{4}$ Landcare Research, Private Bag 3127, Hamilton 3240 New Zealand \\ ${ }^{5}$ Institute of Entomology, Czech Academy of Sciences, Branišovská 31, České Budějovice 37005 Czech Republic
}

Citation: Carmona, C. P., F. de Bello, N. W. H. Mason, and J. Lepš. 2019. Trait probability density (TPD): measuring functional diversity across scales based on TPD with R. Ecology 100(12):e02876. 10.1002/ecy. 2876

Abstract. Functional diversity (FD) has the potential to address many ecological questions, from impacts of global change on biodiversity to ecological restoration. There are several methods estimating the different components of FD. However, most of these methods can only be computed at limited spatial scales and cannot account for intraspecific trait variability (ITV), despite its significant contribution to FD. Trait probability density (TPD) functions (which explicitly account for ITV) reflect the probabilistic nature of niches. By doing so, the TPD approach reconciles existing methods for estimating FD within a unifying framework, allowing FD to be partitioned seamlessly across multiple scales (from individuals to species, and from local to global scales), and accounting for ITV. We present methods to estimate TPD functions at different spatial scales and probabilistic implementations of several FD concepts, including the primary components of FD (functional richness, evenness, and divergence), functional redundancy, functional rarity, and solutions to decompose beta FD into nested and unique components. The TPD framework has the potential to unify and expand analyses of functional ecology across scales, capturing the probabilistic and multidimensional nature of FD. The $\mathrm{R}$ package TPD (https://CRAN.R-project.org/package=TPD) will allow users to achieve more comparative results across regions and case studies.

Key words: functional diversity; functional trait; niche; probability density function; redundancy; uniqueness.

\section{INTRODUCTION}

Functional diversity (FD; the variation of traits in functional space, Carmona et al. 2016a) encompasses several components that can be considered at different scales, from individuals to the global scale. The multitude of existing methods to quantify its different aspects reflects this conceptual complexity (Schleuter et al. 2010, Pavoine and Bonsall 2011). Recently, we proposed a unified framework integrating these different components at any scale (Carmona et al. 2016a, b, 2017). This framework implements the Hutchinsonian (Hutchinson

Manuscript received 14 March 2019; revised 22 July 2019; accepted 30 July 2019. Corresponding Editor: Daniel C. Laughlin.

${ }^{6}$ E-mail: perezcarmonacarlos@gmail.com
1957) concept of the niche as a probabilistic hypervolume (Blonder 2018).

The Hutchinsonian niche (the multidimensional hypervolume in which a species can maintain a population) was originally conceived as a uniform part of the environmental space characterized mainly by its boundaries. However, the performance of the individuals of a species usually varies within niche boundaries; this can be captured by using probability density functions to represent niches. The same concept can be applied to trait distributions of species, reflecting that some trait values are more likely than others. Although most FD methods consider a single value for each species and trait (Villéger et al. 2008, Laliberte et al. 2010, Mouchet et al. 2010, Violle et al. 2017), the concept of trait probability density (TPD) reflects the unequal probabilities of different trait values or combinations of them (Carmona et al. 2016a). Here, we present TPD, an $\mathrm{R}$ 
package implementing this framework. TPD allows users to estimate several components of FD for single or multiple traits, and to partition it seamlessly across multiple scales.

\section{A Probabilistic Framework for Functional Diversity}

Our framework relies on probability density functions, which reflect the chances of observing some specific value for a variable. We refer to these functions as trait probability distributions (TPD). The approach is based on a series of steps that allow estimation of FD at different scales. First, consider a functional space composed of one or more continuous traits, $x$. For each species or population $(S)$ its TPD $\left(T P D_{S}\right)$ can be calculated using different procedures; the TPD package uses kernel density estimation (KDE). Given a set of $\mathrm{n}$ observations of $D$ traits measured in a population of one species $\left(X_{1}, X_{2}, \ldots, X_{n}\right)$, the KDE of the population $\left.\operatorname{(TPD}_{S}(x)\right)$ is:

$$
\operatorname{TPD}_{S}(x ; \mathbf{H})=n^{-1} \sum_{i=1}^{n} K_{\mathbf{H}}\left(x-X_{i}\right)
$$

where $x=\left(x_{1}, x_{2}, \ldots, x_{D}\right)^{T}$ is a D-dimensional trait space, and $X_{i}=\left(X_{i 1}, X_{i 2}, \ldots, X_{i \mathrm{D}}\right)^{T}, i=1,2, \ldots, n . K$ $(x)$, a symmetric probability function, is the kernel (the normal distribution in the TPD package). Although other kernel functions are possible, and implemented elsewhere (Blonder et al. 2014), Gaussian kernels are considered to be the most appropriate alternative for functional diversity applications (Blonder 2018). Finally, $\mathbf{H}$ is the bandwidth matrix, which is symmetrical and positive definite. The TPD package ( $T P D_{S}$ function) uses the unconstrained bandwidth matrix implemented in the R package $k s$ (Duong 2014). This implementation is able to take into account intraspecific trait covariation, an important aspect in some applications of traitbased ecology (Laughlin and Messier 2015).

The number of points needed to characterize the underlying probability density function accurately increases exponentially with the numbers of dimensions considered (Blonder 2018). Hence, the construction of $\mathrm{TPD}_{S}$ functions using KDE procedures for multiple traits may require a prohibitively large amount of trait data. Alternatively, the TPDsMean function allows for the calculation of $\mathrm{TPD}_{S}$ functions when only the mean trait values of species are known, which is often the case when working with data from databases. This is done by estimating the $\mathrm{TPD}_{S}$ function of each species as multivariate normal distributions, using the average and variance of the traits of each species. This procedure requires a previous estimation of the variance assigned to each species, for which there are different alternatives that we summarize in Appendix S1. Regardless of the approach, the resulting $\mathrm{TPD}_{S}$ are probability density functions; hence

$$
\int_{-\infty}^{\infty} \operatorname{TPD}_{S}(x) d x=1 .
$$

For practical reasons in the computation, it is preferable to divide the functional space into a D-dimensional grid composed of many equal-sized cells, with the value of $\mathrm{TPD}_{S}$ being calculated separately for each cell. Accordingly, Eq. 2 would be expressed as

$$
\sum_{i=1}^{N} V \times \mathrm{TPD}_{S_{i}}=1
$$

where $V$ is the size of each of the $N$ cells composing the grid (hypervolume estimated as the product of the edges of the cells), and $\mathrm{TPD}_{S_{i}}$ is the value of the TPD function estimated in the $i$ th cell. The TPD package performs calculations based on these cells. We include the option to define a quantile threshold (Blonder et al. 2014) indicating the proportion of the probability density function of each species or population that will be included in the resulting $\mathrm{TPD}_{S}$, thus reducing the effect of outliers. After thresholding, $\mathrm{TPD}_{S}$ functions are finally rescaled so that the summed probabilities across cells add up to 1 .

Once the $\mathrm{TPD}_{S}$ functions are estimated, the next stage involves combining them to obtain TPD estimates at larger scales (e.g., to estimate the TPD of a community from its constituent species, or to estimate the TPD of a species combining different populations). For instance, the $\mathrm{TPD}_{S}$ of the species in a community can be weighted according to the species' relative abundance. We can then sum the abundance-weighted TPDs to obtain the probability density function of the community $\left(\mathrm{TPD}_{\mathrm{C}}\right)$ using the $T P D_{C}$ function in the package. In weighting each $\mathrm{TPD}_{S}$ by species relative abundance, we ensure that the sum of $\mathrm{TPD}_{\mathrm{C}}$ equals 1 . In this way, the value of $\mathrm{TPD}_{\mathrm{C}}$ for each combination of trait values integrates both the probability distributions of species in trait space and the relative abundances of species in communities, and is thus directly proportional to the relative abundance of that trait combination in the community. We want to underscore that trait values measured in the specific context of interest can be used. For example, when estimating the $\mathrm{TPD}_{\mathrm{C}}$ of a set of communities, it is possible to use for each species and community the trait values of the individuals measured in those particular conditions if that information is available (Carmona et al. 2015b). This way the TPD approach can be useful to compare hypotheses regarding the effects of character displacement (whereby species tend to have other trait values when co-occurring with certain other species). This procedure can be repeated indefinitely to define TPD functions for any spatial scale. For instance, multiple $\mathrm{TPD}_{\mathrm{C}}$ could be combined to obtain a regional TPD.

The TPD package includes functions to characterize several aspects of FD at different scales for single or multiple traits (up to four traits in the current 
implementation). Here, we have arranged the methods according to whether they are applied only (1) within units, regardless of whether these are populations, communities, or regions; or (2) between units, simultaneously considering more than one unit.

\section{Analyses within units}

Some studies focus on describing features related to the distribution of trait values within the unit of interest, such as the amount of functional volume occupied, the evenness in the distribution of abundance in trait space, or the probability associated with each trait value. We include in this group the three components of FD (Mason et al. 2005, Villéger et al. 2008), functional redundancy, and simulations of trait values for predicting species distributions along gradients. Note that although we use examples based on communities, all these procedures could be applied at any scale (populations, communities, regions).

Existing methods to estimate functional richness, evenness, and divergence (Villéger et al. 2008) rely on a single trait value per species, therefore deviating from the original probabilistic definition of these concepts (Mason et al. 2005). The TPD function REND returns to this probabilistic conception.

Functional richness. - Functional richness (FRic) is the amount of functional space occupied by a community. FRic is simply the sum of the hypervolumes of cells in which TPD is greater than 0 (after applying the selected threshold; see the foregoing), and is therefore independent of species abundances. FRic is conceptually similar to the volume yielded by the hypervolume method (Blonder et al. 2014, Carmona et al. 2016b).

Functional evenness.-Functional evenness (FEve) is an indicator of evenness in the distribution of abundance within occupied trait space. We propose an index based on Bulla's (1994) $O$ index for species evenness, later adapted for traits by Mouillot et al. (2005a) and Villéger et al. (2008). We define functional evenness (FEve) as the overlap between the $\mathrm{TPD}_{\mathrm{C}}$ of the considered community and a hypothetical trait distribution occupying the same functional volume with uniform probabilities throughout:

$$
\mathrm{FEve}=\sum_{i=1}^{N} \min \left(V \times \operatorname{TPD}(x=i), V \times N^{-1}\right)
$$

where $V$ represents the size of each cell, and $N$ represents the number of cells occupied by the $\mathrm{TPD}_{\mathrm{C}}$ in question. FEve is close to 1 when all trait values (cells) have similar probabilities, and close to 0 when the majority of total density is concentrated within a few cells.

Functional divergence.-Functional divergence (FDiv) reflects the distribution of abundances within the functional trait volume. Specifically, it measures to what extent trait values near the center of the functional trait volume are more or less dense than trait values at the extremes. We propose a method analogous to (Villéger et al. 2008), but with calculations based on the relative abundance of individual cells within the TPD. Thus, FDiv can be calculated for populations, species and regions, in addition to communities. First, traits are standardized to a $0-1$ scale, making FDiv scale independent. Then the coordinates of the center of gravity$G_{V}=\left(\mathrm{G}_{V 1}, \mathrm{G}_{V 2}, \ldots, \mathrm{G}_{V D}\right)$-of the functional volume occupied by the community are calculated, followed by calculation of the distance of each cell to $G_{V}$ :

$$
d G_{i}=\sqrt{\sum_{k=1}^{D}\left(x_{i k}-g_{k}\right)^{2}}
$$

and the mean distance of all the $N$ cells of the grid in which $\mathrm{TPD}_{\mathrm{C}}$ is greater than 0 :

$$
\overline{d G}=\frac{1}{R} \sum_{i=1}^{N} d G_{i}
$$

The next step consists of determining the sum of relative abundance-weighted deviances $(\Delta d)$ and absolute abundance-weighted deviances $(\Delta|d|)$ for distances from the center of gravity for the $N$ cells:

$$
\begin{aligned}
& \Delta d=\sum_{i=1}^{N} V \times \operatorname{TPD}_{\mathrm{C}_{i}} \times\left(d G_{i}-\overline{d G}\right) \\
& \Delta|d|=\sum_{i=1}^{N} V \times \operatorname{TPD}_{\mathrm{C}_{i}} \times\left|d G_{i}-\overline{d G}\right|
\end{aligned}
$$

where $\mathrm{TPD}_{\mathrm{C}_{i}}$ is analogous to the relative abundances of species in the original method. The absolute deviances represent an upper limit for relative deviances so that an index of functional divergence bound between 0 and 1 (FDiv) can be obtained:

$$
\mathrm{FDiv}=\frac{\Delta d+\overline{d G}}{\Delta|d|+\overline{d G}}
$$

FDiv approaches 0 when the most abundant trait values are close to $G_{V}$, (i.e., $\Delta d$ approaches 0 ) and approaches 1 when the most abundant trait values are those furthest away from $G_{V}$ (i.e., $\Delta d$ approaches $\left.\Delta|d|\right)$.

Functional redundancy.-Two species can be deemed as functionally redundant if they have the same trait values (i.e., if they occupy a similar portion of functional space). Accordingly, removing a highly functionally redundant species from a community should not substantially reduce the community's functional richness. The $\mathrm{R}$ function redundancy yields the average functional redundancy of a community (Carmona et al. 2016a). 
The rationale is simple: for each of the $N$ cells of the grid, count the number of species $(M)$ whose $\mathrm{TPD}_{S}$ value is greater than zero. Functional redundancy is defined as

$$
\mathrm{FRed}=\left(\sum_{i=1}^{N} M_{i} \times V \times \mathrm{TPD}_{\mathrm{C}_{i}}\right)-1
$$

Basically, the method counts how many species display each trait value, and calculates a weighted average of that number, using the probability of that trait in the community - the value of $\mathrm{TPD}_{\mathrm{C}}$ in the cell — as a weighting factor. In this way, redundancy decreases when the most abundant species are more functionally unique (i.e., the most abundant trait values are held by a single species), or when all species become increasingly functionally unique (i.e., all trait values are only held by a single species). After subtracting 1, FRed expresses the abundance-weighted average number of species that could be removed from the community without reducing its functional volume. FRed will approach 0 as species become increasingly functionally unique (or as the most abundant species become functionally unique), and to $S$ - 1 as the $S$ species become increasingly functionally identical. Redundancy defined this way is trivially linked to species richness because its upper bound is $S-1$ (in the case that all species are functionally identical, all but one species could go extinct from a community without altering its functional structure). A simple solution to break this relationship is to divide redundancy values by $S-1$, so that redundancy is expressed in relative terms and bounded between 0 and 1 . This allows comparisons of communities with different numbers of species (see Appendix S2 for more details on this correction).

Trait simulations. - Some functional trait-based applications require drawing trait values from TPD distributions (e.g., the Traitspace model; Laughlin et al. 2012, 2015). The $\mathrm{R}$ function $t$ Samp uses TPD functions to draw these trait values randomly at any scale.

\section{Analyses between units}

The following methods help in understanding the mechanisms driving differences in functional structure between populations, communities, and regions ( $\beta$ functional diversity).

Overlap-based functional dissimilarity $\left(\beta_{O}\right)$. - As with classic dissimilarity metrics for turnover in species composition (i.e., Jaccard and Sorensen-Dice), functional dissimilarity between two communities can be calculated by comparing the proportion of joint density distributions $(A)$ to the proportion unique to either community ( $B$ and $C$ ). For two communities, $i$ and $j$, we estimate $A$ (overlap) in a way that is analogous to that proposed by (Mouillot et al. 2005b) for a single trait dimension:

$$
A=\sum_{i=1}^{N} \min \left(V \times \operatorname{TPD}_{\mathrm{C}_{i}}, V \times \operatorname{TPD}_{\mathrm{C}_{j}}\right)
$$

and because both $\mathrm{TPD}_{\mathrm{C}}$ functions sum to 1 :

$$
B=C=1-A .
$$

The dissimilarity between the two communities $\left(\beta_{\mathrm{O}}\right)$ can then be estimated as 1 minus the overlap (Lepš et al. 2006), or, equivalently:

$$
\beta_{\mathrm{O}}=\frac{B+C}{2 A+B+C} .
$$

$\beta_{\mathrm{O}}$ (function dissim) is bounded between 0 , when two units are functionally identical $(A=1, B=C=0)$, and 1 , when there is no functional overlap between them $(A=0, B=C=1)$.

Functional rarity.-One of the most interesting features of TPD functions is that they are conceptually scale independent. Effectively, this means that the TPD of a given ecological unit (e.g., a population) can be compared with the TPD of units from disparate scales (e.g., with communities or regions). This allows estimation of $\beta_{\mathrm{O}}$ also between units at different hierarchical levels. This way, by comparing the $\mathrm{TPD}_{S}$ functions of individual species to those of the local or regional pool of species, we can obtain a measure of the functional distinctiveness of each species within the community or region, or of each community within a region (function uniqueness; Carmona et al. 2017).

Decomposition of functional dissimilarity.-Taxonomic dissimilarity between communities can be decomposed into two components: turnover (because of species replacement) and nestedness (because of differences in species richness; Baselga 2010). This decomposition was extended to functional dissimilarity, using the communities' convex hull-based hypervolumes (Villéger et al. 2013). However, convex hulls are sensitive to outliers and are not able to detect gaps (or "holes") in the occupation of functional space (Podani 2009, Blonder 2016, Carmona et al. 2016a). We present a method to decompose functional dissimilarity into two complementary components while avoiding the shortcomings of convex hulls (function dissim). One of the components results from the difference in density values between $\mathrm{TPD}_{\mathrm{C}_{i}}$ and $\mathrm{TPD}_{\mathrm{C}_{j}}$ within the shared functional volume $\left(P_{\mathrm{N}}\right)$; the remainder is the functional volume unique to each community $\left(P_{\mathrm{U}}\right)$. Calculating these components is relatively simple. Using the same nomenclature as above, $B$ (and also $C$ ) can be further decomposed into two components: " $B_{\mathrm{N}}$ " is the proportion of $B$ within cells occupied by both communities (i.e., the part of $B$ that is "above" $A$ ), and " $B_{\mathrm{U}}$ " is the proportion of $B$ contributed by cells uniquely occupied by community $i$ 
(Carmona et al. 2016a). Following Villéger et al. (2013),

$$
\begin{gathered}
P_{\mathrm{U}}=\frac{2 \min \left(B_{\mathrm{U}}, C_{\mathrm{U}}\right)}{B_{\mathrm{N}}+C_{\mathrm{N}}+2 \min \left(B_{\mathrm{U}}, C_{\mathrm{U}}\right)} \\
P_{\mathrm{N}}=\frac{B_{\mathrm{N}}+C_{\mathrm{N}}}{B_{\mathrm{N}}+C_{\mathrm{N}}+2 \min \left(B_{\mathrm{U}}, C_{\mathrm{U}}\right)} .
\end{gathered}
$$

High levels of functional dissimilarity $\left(\beta_{\mathrm{O}}\right)$ between units of the same hierarchical level can be the result of different processes. For example, two communities can be very dissimilar because the species of each one occupy different parts of functional space; in this case $P_{\mathrm{U}}$ will account for the greatest proportion of dissimilarity. On the other hand, if the high dissimilarity is mostly because the functional volume of one is completely nested within the functional volume of the other, $P_{\mathrm{N}}$ will approach 1. Thus, $P_{\mathrm{U}}$ and $P_{\mathrm{N}}$ are broadly analogous to the turnover and nestedness components of beta diversity, respectively.

\section{Case Study: Functional Diversity Patterns Along an Environmental Gradient in a Mediterranean GRASSLAND}

We illustrate the TPD framework using data from a Mediterranean annual grassland located $20 \mathrm{~km}$ north of Madrid, in central Spain $\left(40^{\circ} 36^{\prime} \mathrm{N}, 3^{\circ} 45^{\prime} \mathrm{W}\right.$; elevation $\sim 700 \mathrm{~m}$ ). We selected a topographical gradient encompassing substantial differences in productivity within a limited spatial scale (described in more detail in Carmona et al. 2015b). The gradient runs from the upper slope downhill with an inclination of $15^{\circ}$. Previous studies on similar slopes in the same area have described substantial differences in soil water availability, clay percentage, total nitrogen, and soil organic matter content associated with the position on the slope (Peco et al. 2006), which are finally reflected in a much higher productivity of lower-slope areas (Carmona et al. 2013). In spring 2013, we placed $4020 \times 20 \mathrm{~cm}$ quadrats in a line, at distances of $2 \mathrm{~m}$ from each other. In each quadrat, we recorded species cover, and collected 10 individuals of the most abundant species, and in doing so we accounted for at least $90 \%$ of the cover of the quadrat. For these selected individuals, we measured two widely used functional traits: plant height $(\mathrm{cm})$ and specific leaf area $\left(\mathrm{SLA} ; \mathrm{mm}^{2} / \mathrm{mg}\right)$. This sampling strategy gave us data at the population level (species within quadrats, for a total of 254 populations of 51 species). Measurements of soil water content in the studied quadrats revealed that soil water content increased downhill in a linear fashion (Appendix S3: Fig. S1). Further information on the sampling site and methods can be found in Carmona et al. (2015b). With the traits measured at the individual level, we calculated the different indices outlined above for single and multiple traits. We used the kernel density estimator approach to calculate a separate $\mathrm{TPD}_{S}$ function for each population of each species (as represented by 10 individuals within a quadrat). In this section we present a small selection of results, considering two different scales: the community scale, using the $\mathrm{TPD}_{\mathrm{C}}$ functions of the different quadrats, and the species scale, using the $\mathrm{TPD}_{S}$ functions of populations of Plantago lagopus, the species that occurred most frequently in the quadrats.

We studied changes in FRic in relation to soil water content, both at the population and community levels. FRic was lowest at the driest end of the gradient (i.e., uphill), both for individual and multiple traits, and both for communities and P. lagopus populations. Interestingly, communities and populations with smaller functional volumes of SLA tended to have smaller functional volumes of height too (Fig. 1a). This suggests that environmental filtering processes reducing the range of trait values operate simultaneously on both traits (Cornwell et al. 2006; Fig. 1a), and at both the community and species level. For populations, FRic increased linearly along with soil water content for height and both height and SLA together, but reached its maximum at intermediate water contents for SLA (Fig. 1a). This shows that the predominant processes along environmental gradients can differ between traits, emphasizing that it is often necessary to analyze functional diversity patterns considering individual traits separately (Mason et al. 2012, Spasojevic and Suding 2012, Carmona et al. 2015a). Next we analyzed the decay of functional similarity with distance (Lamanna et al. 2014) in populations and communities, and its decomposition. We calculated $\beta_{\mathrm{O}}$ and $P_{\mathrm{N}}$ for each pair of populations or communities, and plotted them against the distance between the quadrats in the gradient. We performed Mantel tests (999 repetitions) between the respective distance matrices to see if the relationships were significant. The relationship between functional dissimilarity and spatial distance clearly showed that using $\beta_{\mathrm{O}}$ allowed us to detect functional differences between populations and communities. Communities and populations at the opposite extremes of the gradient showed high functional differentiation, especially when considering height and SLA simultaneously (Fig. 1b). In this sense, the populations of $P$. lagopus displayed a remarkable level of ITV across a very short spatial gradient, with almost no functional overlap between the populations of the highest and lowest parts of the slope. These results emphasize that disregarding ITV - by considering only average trait values of species - may result in inadequate characterizations of the functional structure of communities (Carmona et al. 2015b). In general, the proportion of dissimilarity due to differences in the abundance of shared traits $\left(P_{\mathrm{N}}\right)$ decreased with spatial distance as dissimilarity increased (Fig. 1b). This suggests that spatially distant communities and populations occupy increasingly different regions of functional space. However, regarding SLA of communities, $P_{\mathrm{N}}$ was very high regardless of spatial distance between communities. This suggests that the range of 
(a) Within units Plantago lagopus
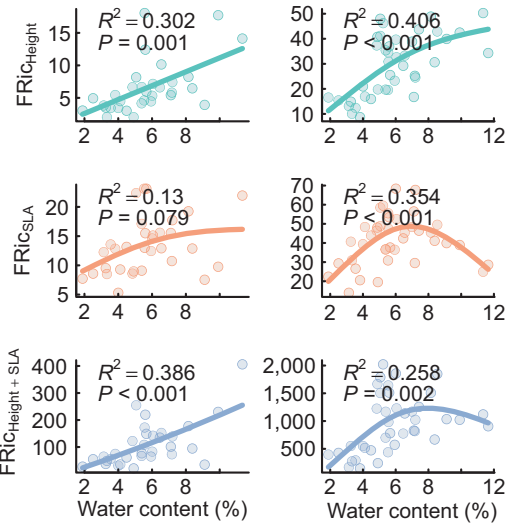

(c) Redundancy vs. richness

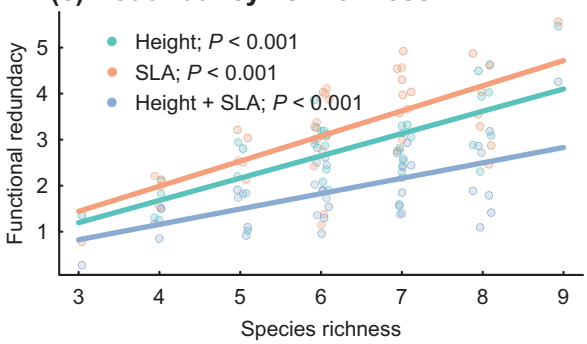

\section{(b) Between units}
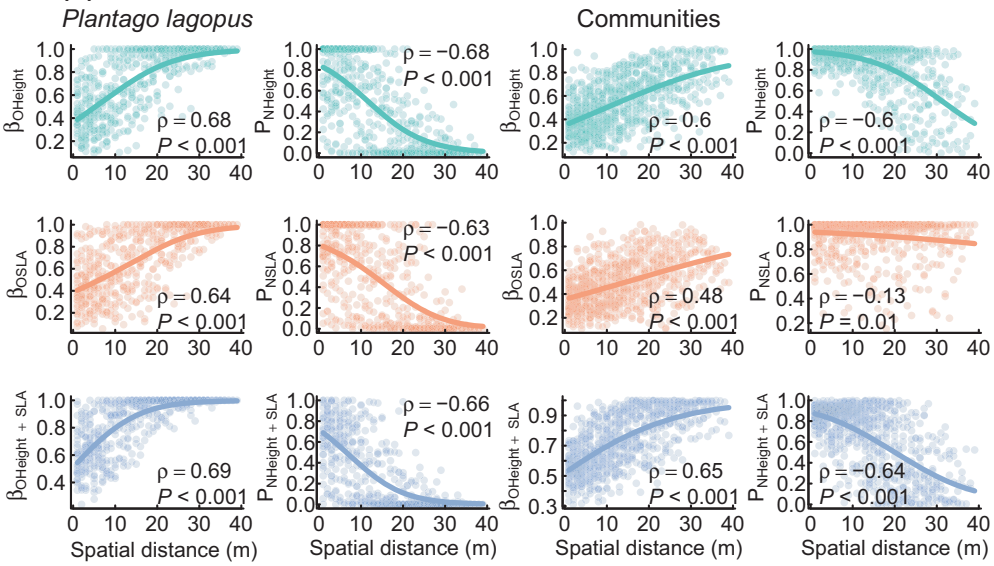

(d) Relative redundancy

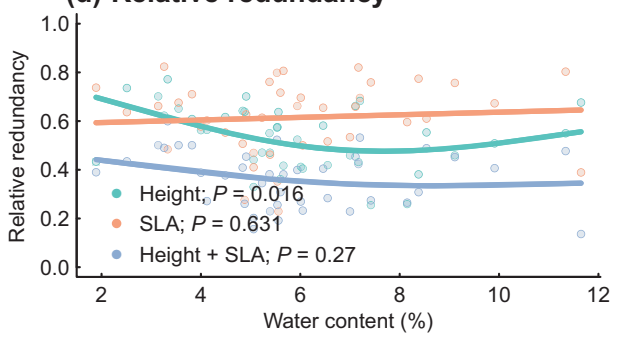

FIG. 1. Application of the framework in a Mediterranean annual grassland. (a) Relationship between the FRic values of communities, calculated using single (specific leaf area [SLA] or Height) or multiple (SLA and Height) traits, and soil water content. (b) Relationship between functional dissimilarity $\left(\beta_{\mathrm{O}}\right)$ and the proportion of dissimilarity due to differences in shared trait volume $\left(P_{\mathrm{N}}\right)$ with topographical distance for populations of Plantago lagopus and communities, for single and multiple traits. Each plot includes the correlation between the two distances $(\rho)$, the significance of Mantel test, and a lowess regression to illustrate the relationship. (c) Relationship between species richness and functional redundancy in each quadrat, calculated for single and multiple traits. (d) Changes in functional redundancy (expressed in relative terms) for single and multiple traits along the soil water content gradient.

SLA values of those communities occupying a smaller functional volume was a subset of that of communities with greater values of FRic (Fig. 1b).

We also examined the relationship between functional redundancy - considering single and multiple traitsand species richness in communities. We checked whether this relationship was linear or nonlinear by fitting gam models, setting an upper limit of three degrees of freedom associated with the smooth estimation. We found no evidence of nonlinear relationships between redundancy and species richness in our dataset. Consistent with the notion that redundancy should decrease as more traits are considered (Rosenfeld 2002), redundancy was lower in the multidimensional case (Fig. 1c). In addition, the interpretation of the relationship between species richness and redundancy is straightforward with our method; redundancy increased with species richness, reflecting the idea that species-rich communities have a higher degree of functional overlap between species (Fig. 1c).

We also analyzed how relative redundancy varied along the water content gradient by applying the S-1 correction. Our results revealed that redundancy in height was greater at the driest end of the gradient, and decreased in a nonlinear fashion as water availability increased (Fig. 1d). This result is most likely due to the overabundance of small individuals when water availability is low. When water availability increases, the coexistence of short and tall individuals is possible, resulting in decreased redundancy (as also indicated by higher FRic levels).

\section{Conclusions}

In the context of rapid environmental change and associated losses of species, it is essential to predict changes in species composition and ecosystem functioning from local through to global levels. This requires the development of standardized tools to characterize these impacts. The TPD framework (with its future extensions) has emerged as an alternative with great potential to tackle several questions in ecology; for example, identifying changes in the functional divergence and evenness within communities (Botta-Dukát and Czúcz 2016), 
indicating reductions in the relative abundance of species or individuals with trait values sensitive to disturbances, can help to anticipate local extinctions - and their associated reductions in functional richness-before these extinctions take place (Mason et al. 2013). The precise quantification of redundancy that we present here may help to answer questions regarding what levels of functional redundancy are needed to ensure the capacity of ecosystems to recover after perturbations (Walker 1995). Redundancy can also be used in combination with nested subset analyses to estimate the vulnerability of ecosystem function to species loss (Zavaleta and Hulvey 2004, Sasaki et al. 2014, Carmona et al. 2017). Combining information on redundancy and overlap-based dissimilarity between species and communities appears to be a promising approach in aiding the design of ecological restoration projects, including those intended to exclude invasive species (Laughlin 2014), or for the protection of species supporting specific ecosystem functions (Mouillot et al. 2013). Indeed, although the TPD framework, as presented here, is primarily explained in terms of functional niche, similar concepts could be applied to other types of data, such as isotope ratios (Swanson et al. 2015), climatic data (Blonder et al. 2014), or habitat preferences (Traba et al. 2015, 2017), as well as to traits of other taxonomic groups (Martello et al. 2018); these are only some examples, but the number of potential applications of the framework is considerable. With this in mind, we want to emphasize that we do not consider the TPD framework to be a definitive and closed collection of methods, but rather the first step towards a unified framework that accommodates the probabilistic and multidimensional nature of the functional facet of diversity. We hope that the inclusion of these methods in the toolbox of ecologists will help improve our ability to predict and understand the consequences of environmental changes on ecosystems.

\section{ACKNOWLEDGMENTS}

CPC was supported by the Estonian Research Council (project PSG293), and by the European Union through the European Regional Development Fund (Centre of Excellence EcolChange).

\section{Literature Cited}

Baselga, A. 2010. Partitioning the turnover and nestedness components of beta diversity. Global Ecology and Biogeography 19:134-143.

Blonder, B. 2016. Do hypervolumes have holes? American Naturalist 187:E93-E105.

Blonder, B. 2018. Hypervolume concepts in niche- and traitbased ecology. Ecography 41:1441-1455.

Blonder, B., C. Lamanna, C. Violle, and B. J. Enquist. 2014. The n-dimensional hypervolume. Global Ecology and Biogeography 23:595-609.

Botta-Dukát, Z., and B. Czúcz. 2016. Testing the ability of functional diversity indices to detect trait convergence and divergence using individual-based simulation. Methods in Ecology and Evolution 7:114-126.
Bulla, L. 1994. An index of evenness and its associated diversity measure. Oikos 70:167-171.

Carmona, C. P., F. de Bello, N. W. H. Mason, and J. Leps. 2016a. Traits without borders: integrating functional diversity across scales. Trends in Ecology \& Evolution 31:382-394.

Carmona, C. P., F. de Bello, N. W. H. Mason, and J. Lep̌s. 2016b. The density awakens: a reply to Blonder. Trends in Ecology and Evolution 31:667-669.

Carmona, C. P., F. de Bello, T. Sasaki, K. Uchida, and M. Pärtel. 2017. Towards a common toolbox for rarity: a response to Violle et al.. Trends in Ecology and Evolution 32:889-891.

Carmona, C. P., N. W. H. Mason, F. M. Azcárate, and B. Peco. 2015a. Inter-annual fluctuations in rainfall shift the functional structure of Mediterranean grasslands across gradients of productivity and disturbance. Journal of Vegetation Science 26:538-551.

Carmona, C. P., A. Röder, F. M. Azcárate, and B. Peco. 2013. Grazing management or physiography? Factors controlling vegetation recovery in Mediterranean grasslands. Ecological Modelling 251:73-84.

Carmona, C. P., C. Rota, F. M. Azcárate, and B. Peco. 2015 b. More for less: sampling strategies of plant functional traits across local environmental gradients. Functional Ecology 29:579-588.

Cornwell, W. K., D. W. Schwilk, and D. D. Ackerly. 2006. A trait-based test for habitat filtering: convex hull volume. Ecology 87:1465-1471.

Duong, T. 2014. ks: Kernel smoothing. R package version 1.9.2. https://cran.r-project.org/web/packages/ks/index.html

Hutchinson, G. E. 1957. Concluding remarks. Cold Spring Harbor Symposia on Quantitative Biology 22:415-427.

Laliberte, E., P. Legendre, S. Ecology, and N. January. 2010. A distance-based framework for measuring functional diversity from multiple traits. Ecology 91:299-305.

Lamanna, C., et al. 2014. Functional trait space and the latitudinal diversity gradient. Proceedings of the National Academy of Sciences USA 111:13745-13750.

Laughlin, D. C. 2014. Applying trait-based models to achieve functional targets for theory-driven ecological restoration. Ecology Letters 17:771-784.

Laughlin, D. C., C. Joshi, S. J. Richardson, D. A. Peltzer, N. W. H. Mason, and D. A. Wardle. 2015. Quantifying multimodal trait distributions improves trait-based predictions of species abundances and functional diversity. Journal of Vegetation Science 26:46-57.

Laughlin, D. C., C. Joshi, P. M. van Bodegom, Z. A. Bastow, and P. Z. Fulé. 2012. A predictive model of community assembly that incorporates intraspecific trait variation. Ecology Letters 15:1291-1299.

Laughlin, D. C., and J. Messier. 2015. Fitness of multidimensional phenotypes in dynamic adaptive landscapes. Trends in Ecology \& Evolution 30:487-496.

Lepš, J., F. de Bello, S. Lavorel, and S. Berman. 2006. Quantifying and interpreting functional diversity of natural communities: practical considerations matter. Preslia 78: 481-501.

Martello, F., F. De Bello, M. S. De Castro Morini, R. R. Silva, D. R. De Souza-Campana, M. C. Ribeiro, and C. P. Carmona. 2018. Homogenization and impoverishment of taxonomic and functional diversity of ants in Eucalyptus plantations. Scientific Reports 8:3266.

Mason, N. W. H., F. de Bello, D. Mouillot, S. Pavoine, and S. Dray. 2013. A guide for using functional diversity indices to reveal changes in assembly processes along ecological gradients. Journal of Vegetation Science 24:794-806. 
Mason, N. W. H., D. Mouillot, W. G. Lee, and J. B. Wilson. 2005. Functional richness, functional and functional evenness divergence: the primary of functional components diversity. Oikos 111:112-118.

Mason, N. W. H., S. J. Richardson, D. A. Peltzer, F. de Bello, D. A. Wardle, and R. B. Allen. 2012. Changes in coexistence mechanisms along a long-term soil chronosequence revealed by functional trait diversity. Journal of Ecology 100:678-689.

Mouchet, M. A., S. Villéger, N. W. H. Mason, and D. Mouillot. 2010. Functional diversity measures: an overview of their redundancy and their ability to discriminate community assembly rules. Functional Ecology 24:867-876.

Mouillot, D., et al. 2013. Rare species support vulnerable functions in high-diversity ecosystems. PLoS Biology 11:e1001569.

Mouillot, D., N. W. H. Mason, O. Dumay, and J. B. Wilson. $2005 a$. Functional regularity: a neglected aspect of functional diversity. Oecologia 142:353-359.

Mouillot, D., W. Stubbs, M. Faure, O. Dumay, J. A. Tomasini, J. B. Wilson, and T. Do Chi. 2005b. Niche overlap estimates based on quantitative functional traits: a new family of nonparametric indices. Oecologia 145:345-353.

Pavoine, S., and M. B. Bonsall. 2011. Measuring biodiversity to explain community assembly: a unified approach. Biological Reviews 86:792-812.

Peco, B., A. M. Sánchez, and F. M. Azcárate. 2006. Abandonment in grazing systems: consequences for vegetation and soil. Agriculture, Ecosystems \& Environment 113:284-294.

Podani, J. 2009. Convex hulls, habitat filtering, and functional diversity: mathematical elegance versus ecological interpretability. Community Ecology 10:244-250.

Rosenfeld, J. S. 2002. Functional redundancy in ecology and conservation. Oikos 98:156-162.

Sasaki, T., M. Katabuchi, C. Kamiyama, M. Shimazaki, T. Nakashizuka, and K. Hikosaka. 2014. Vulnerability of moorland plant communities to environmental change: consequences of realistic species loss on functional diversity. Journal of Applied Ecology 51:299-308.

Schleuter, D., M. Daufresne, F. Massol, and C. Argillier. 2010. A user's guide to functional diversity indices. Ecological Monographs 80:469-484.

Spasojevic, M. J., and K. N. Suding. 2012. Inferring community assembly mechanisms from functional diversity patterns: the importance of multiple assembly processes. Journal of Ecology 100:652-661.

Swanson, H. K., M. Lysy, M. Power, A. D. Stasko, J. D. Johnson, and J. D. Reist. 2015. A new probabilistic method for quantifying $\mathrm{n}$-dimensional ecological niches and niche overlap. Ecology 96:318-324.

Traba, J., E. C. Iranzo, C. P. Carmona, and J. E. Malo. 2017. Realised niche changes in a native herbivore assemblage associated with the presence of livestock. Oikos 126:1400-1409.

Traba, J., M. B. Morales, C. P. Carmona, and M. P. Delgado. 2015. Resource partitioning and niche segregation in a steppe bird assemblage. Community Ecology 16:178-188.

Villéger, S., G. Grenouillet, and S. Brosse. 2013. Decomposing functional $\beta$-diversity reveals that low functional $\beta$-diversity is driven by low functional turnover in European fish assemblages. Global Ecology and Biogeography 22:671-681.

Villéger, S., N. W. H. Mason, and D. Mouillot. 2008. New multidimensional functional diversity indices for a multifaceted framework in functional ecology. Ecology 89:2290-22301.

Violle, C., W. Thuiller, N. Mouquet, F. Munoz, N. J. B. Kraft, M. W. Cadotte, S. W. Livingstone, and D. Mouillot. 2017. Functional rarity: the ecology of outliers. Trends in Ecology \& Evolution 13:1030-1340.

Walker, B. 1995. Conserving biological diversity through ecosystem resilience. Conservation Biology 9:747-752.

Zavaleta, E. S., and K. B. Hulvey. 2004. Realistic species losses disproportionately reduce grassland resistance to biological invaders. Science 306:1175-1177.

\section{SUPPORTING INFORMATION}

Additional supporting information may be found in the online version of this article at http://onlinelibrary.wiley.com/doi/ 10.1002/ecy.2876/suppinfo 